\title{
Function of basal ganglia in bridging cognitive and motor modules to perform an action
}

\author{
Atsuko Nagano-Saito ${ }^{1,2 *}$, Kristina Martinu ${ }^{1}$ and Oury Monchi ${ }^{1,2}$ \\ ' Centre de Recherche, Institut Universitaire de Gériatrie de Montréal, Montréal, OC, Canada \\ 2 Department of Radiology, Université de Montréal, Montréal, QC, Canada
}

\author{
Edited by: \\ Tobias H. Donner, University of \\ Amsterdam, Netherlands \\ Reviewed by: \\ V. S. Chandrasekhar Pammi, \\ University of Allahabad, India \\ Chung-Chuan Lo, National Tsing \\ Hua University, Taiwan \\ *Correspondence: \\ Atsuko Nagano-Saito, Centre de \\ Recherche de I'Institut Universitaire \\ de Gériatrie de Montréal, 4545 \\ Chemin Queen Mary, Montréal, \\ OC H3W 1W4, Canada \\ e-mail:atsuko.nagano@gmail.com
}

The basal ganglia (BG) are thought to be involved in the integration of multiple sources of information, and their dysfunction can lead to disorders such as Parkinson's disease (PD). PD patients show motor and cognitive dysfunction with specific impairments in the internal generation of motor actions and executive deficits, respectively. The role of the $B G$, then, would be to integrate information from several sources in order to make a decision on a resulting action adequate for the required task. Reanalyzing the data set from our previous study (Martinu et al., 2012), we investigated this hypothesis by applying a graph theory method to a series of fMRI data during the performance of self-initiated (SI) finger movement tasks obtained in healthy volunteers (HV) and early stage PD patients. Dorsally, connectivity strength between the medial prefrontal areas (mPFC) and cortical regions including the primary motor area (M1), the extrastriate visual cortex, and the associative cortex, was reduced in the PD patients. The connectivity strengths were positively correlated to activity in the striatum in both groups. Ventrally, all connectivity between the striatum, the thalamus, and the extrastriate visual cortex decreased in strength in the PD, as did the connectivity between the striatum and the ventrolateral PFC (VLPFC). Individual response time (RT) was negatively correlated to connectivity strength between the dorsolateral PFC (DLPFC) and the striatum and positively correlated to connectivity between the VLPFC and the striatum in the HV. These results indicate that the $B G$, with the $\mathrm{MPFC}$ and thalamus, are involved in integrating multiple sources of information from areas such as DLPFC, and VLPFC, connecting to $M 1$, thereby determining a network that leads to the adequate decision and performance of the resulting action.

Keywords: basal ganglia, dopamine, fMRI, cross-network synchrony, Parkinson's disease

\section{INTRODUCTION}

The basal ganglia (BG), mainly consisting of the striatum, the globus pallidus, the substantia nigra, and the subhalamic nucleus, are involved in a variety of functions. The striatum [caudate, putamen, and ventral striatum (VS)] is the main input of the $\mathrm{BG}$, and the thalamus connects the BG to the cortex through cortico-BG-thalamo-cortical loops and brainstem-thalamo-BGbrainstem loops (Alexander et al., 1986; Albin et al., 1989; McHaffie et al., 2005). The striatum receives extensive glutamatergic afferents from a wide variety of cortical regions (Parent and Hazrati, 1995). The dopaminergic projections from the midbrain to the striatum are a crucial modulator of striatal processing of glutamatergic cortical and thalamic signals on the striatum principal neurons (Surmeier et al., 2009). Patients with Parkinson's

Abbreviations: BG, basal ganglia; PD, Parkinson's disease; HV, healthy volunteers; SI, self-initiated; ET, externally triggered; CTL, control; pre-SMA, presupplemental motor area; SMA, supplemental motor area; M1, primary motor area; $\mathrm{mPFC}$, medial prefrontal cortex; DLPFC, dorsolateral prefrontal cortex; VLPFC, ventrolateral prefrontal cortex; IPL, intra parietal lobe; PMd, dorsal premotor cortex; LG, lingual gyrus; FG, fusiform gyrus; RT, response time; ROI, regions of interest. disease (PD), characterized by motor and cognitive dysfunctions, show significant reduction of the dopaminergic projections to the striatum (Samii et al., 2004). Patients generally display difficulties in performing internally generated movements (Georgiou et al., 1993), and cognitive deficits often consist of decreases in executive functions (Litvan et al., 1991). Internal generation of movement and executive functions both require decision-making processes in order to select an action among several alternative possibilities for the task at hand. The BG, then, mostly modulated by dopaminergic projections, seem to have an important role in the mediation of cognitive and motor modules to generate an appropriate decision on a resulting action for the task being performed.

In the context of brain networks, it is not clear how BG neuro-modulation affects decision making, and how it is impaired by dopaminergic dysfunction. Previous studies indicate that executive deficits in PD are associated with dysfunction of the caudate nucleus in PD patients without dementia (Lewis et al., 2003; Monchi et al., 2004, 2007). This suggests that dopamine is involved in the transfer of information first processed in cognitive brain networks, toward motor-related 
networks, sequentially. Furthermore, PD patients with mild cognitive impairment (MCI), compared with non-MCI patients, showed dysfunction of the caudate nucleus, along with a stronger reduction of activity in the motor cortex, but without any differences in motor-ability (Nagano-Saito et al., 2013). As of now, however, it is not clear how the BG are organized in order to effectively combine cognitive and motor modules to conduct a series of tasks. While performing the Wisconsin Card Sorting Task (WCST), we observed increases in functional connectivity between the frontal regions [including the dorsolateral prefrontal cortex (DLPFC), the ventrolateral prefrontal cortex (VLPFC), and the pre-supplementary motor area (pre-SMA)], and the putamen in healthy volunteers (HV). This effect was diminished with transient dopamine depletion (Nagano-Saito et al., 2008). Moreover, the strength of the connectivity between the DLPFC and the striatum could estimate individual response times (RT) (Nagano-Saito et al., 2008). Another study with self-initiated (SI) movements showed increased functional connectivity between cortical areas (pre-SMA and the primary motor area) and the putamen in HV; this was diminished in PD patients (Wu et al., 2011). Since the DLPFC and the pre-SMA are components of the cognitive network (Alexander et al., 1986; Albin et al., 1989; Picard and Strick, 2001), the resulting fMRI functional connectivity may reflect the combination of both the cognitive and motor modules.

SI finger movements are simple, but require a certain level of decision-making, such as selecting one action among several alternative possibilities. SI movements have been shown to recruit a larger number of brain activations, including the DLPFC, VLPFC, medial prefrontal cortex (mPFC), striatum, and thalamus, compared with single-button presses (FrancoisBrosseau et al., 2009; Martinu et al., 2012). These activations were decreased in PD patients (Martinu et al., 2012). PD patients are impaired in the internal generation of movement (Georgiou et al., 1993; Kiesel et al., 2010), plausibly linking the impairment of movement prediction for task preparation (Werheid et al., 2007). The motor dysfunction in PD patients may be related with the impairment in decision-making processes, like predicting possible actions. Based on animal single cell recordings, decision making properties seem to be expressed by the same cells as sensorimotor processes, joining what should be undertaken by cognitive processes with movement execution, rather than showing localization within particular higher cognitive centers in the brain (Cisek and Kalaska, 2010). In humans, even simple perceptual decisions emerge from recurrent and flexible interactions between widely distributed regions of the cerebral cortex, showing an oscillatory interaction between the regions (Siegel et al., 2011). Considering these finding, the oscillation of interaction (e.g., between the cognitive and motor modules) during decisionmaking processes required for the SI task could be operated by the BG system.

The application of graph theory methods to brain imaging data is a simple and powerful mathematical framework for the characterization of topological features of brain networks (Bullmore and Sporns, 2009; He and Evans, 2010). The methods enable us to investigate the structural features in the brain, including both the local-, and global-level connectivity. In graph theory, a network consists of nodes and edges (connections between any two nodes). The network wiring cost is defined as the ratio of actual connections to the maximum number of possible connections, and the topological features of networks, such as global, local, and cost efficiencies, are known to change as the function of wiring costs change (Latora and Marchiori, 2001; Bullmore and Sporns, 2009; He and Evans, 2010). The global efficiency is an index of inverse path length, defined by an average minimum number of connections that link any two nodes of the network, and indicates the efficiency of information transfer among different brain regions (Achard et al., 2006; He and Evans, 2010). The local efficiency is defined as an average of the clustering coefficients over all nodes in the network, and a measure of local clustering and fault tolerance (Achard et al., 2006; He and Evans, 2010). Functional MRI and MEG resting-state and task-based studies with this method have shown that the human brain has small-world network properties with a certain range of network wiring cost (Achard et al., 2006; Bassett and Bullmore, 2006; Achard and Bullmore, 2007; Bassett et al., 2011; Kitzbichler et al., 2011; Carbonell et al., 2014). This method also unveiled that PD patients decreased local connectivity function in the $\mathrm{mPFC}$, prefrontal cortex and striatum, and globally measured network efficiency indicated lowered information flow in the brain $(\mathrm{Wu}$ et al., 2009; Cao et al., 2011; Skidmore et al., 2011; Baradaran et al., 2013; Gottlich et al., 2013).

A small-world network consists of nodes that are not necessarily neighbors, but can be reached by a small number of steps, displaying an enhanced signal-propagation speed (Watts and Strogatz, 1998). The networks with small-world network properties show greater local efficiency than a random network, and less global efficiency than a random network but still greater than a regular lattice network (Achard et al., 2006; Achard and Bullmore, 2007; Skidmore et al., 2011; Carbonell et al., 2014). The small-world network property is considered as the outcome of a selection by competitive criteria: minimization of wiring cost vs. maximization of efficiency of information transfer (Bassett and Bullmore, 2006; Kitzbichler et al., 2011). Cost efficiency is defined as "global efficiency - cost," and it is assumed that the brain operates optimally with the maximum cost efficiency, maximizing information transfer (Sporns et al., 2000; Achard et al., 2006; Achard and Bullmore, 2007; Bassett et al., 2009).

Intrinsic patterns of functional connectivity of the human brain have been shown in the visual, auditory, motor, taskcontrol, and default mode networks (Biswal et al., 1995; Greicius et al., 2003; Fox et al., 2005; Power et al., 2011), corresponding to relatively high local efficiency (Kitzbichler et al., 2011). The small-world network property hypothesis suggests rapid crossnetwork synchronization, which plays a crucial role in information processing (Lago-Fernandez et al., 2000; Masuda and Aihara, 2004; Bassett et al., 2011). Patterns of the brain network are rapidly modulated by task complexity, showing cross-network synchronization during more complex tasks, possibly supported by relatively high global efficiency of the brain (Kitzbichler et al., 2011). Thus, considering small-world network properties while applying graph theory methods during a task would enable us to study a condition of the global brain connectivity, crucial for the performance of a given task. The graph theory approach also 
helps in investigating the local connectivity one by one, such as between nodes belonging to the cognitive and motor networks, and between nodes belonging to the cortical and subcortical areas.

In the present study, we wanted to explore the hypothesis that the BG play an important role in the integration of cognitive and motor networks in order to proactively perform tasks. We used a previous $\mathrm{fMRI}$ data set from $\mathrm{HV}$ and PD patients performing SI finger movements (Martinu et al., 2012). More specifically, we first investigated economical connectivity considering the maximum cost efficiency with a feature of small-world network during the SI finger movement task in the HV by applying the graph theory methods with a cost-threshold approach. Here, the individual networks were structured with fixed costs, rather than with fixed correlation threshold (the minimal correlation ratio). The cost-threshold approach was applied in order to consider the maximum cost efficiency, and because this approach is more relevant in the analysis of connectivity "patterns" between different populations (HV vs. PD). We then compared this pattern with PD patients' connectivity. In addition, we investigated whether the activity of the striatum in relation to cortico-cortical and cortico-striatal connectivity could estimate individual behavior.

\section{MATERIALS AND METHODS PARTICIPANTS}

Fourteen right-handed HV (mean age 61.74 \pm 6.62 years, six males) and 12 right-handed patients at the early and moderate stages of idiopathic non-demented PD (mean age $62.89 \pm 6.70$ years, six males) were recruited (for detailed patient information, see Martinu et al., 2012). All patients were diagnosed by a movement disorders neurologist and met the United Kingdom Brain Bank criteria for idiopathic PD (Hughes et al., 1992). All patients were in Hoehn-Yahr stage I or II (Hoehn and Yahr, 1967). At the time of fMRI scanning, patients were asked to withdraw from all antiparkinsonian medications at least $12 \mathrm{~h}$ prior to the appointment. All subjects gave written informed consent to the protocol which was reviewed and approved by the research ethics committee of the Regroupement Neuroimagerie Quebec (CMER-RNQ) at the Institut Universitaire de Gériatrie de Montréal, following the guidelines of the Tri-Council Policy Statement of Canada, the civil code of Quebec, the Declaration of Helsinki, and the code of Nuremberg. All the participants who declined to participate or did not participate were not disadvantaged in any other way by not participating in the study. None of the participants had a compromised ability to consent on their own.

\section{BEHAVIORAL TASKS}

Using both their right and left hands separately, participants performed a SI random movement condition, an externally-triggered (ET) follow condition, and a single-button repeat condition as a control (CTL). Five blue squares were displayed, each square corresponding to a button on the response box; all fingers were used except for the little finger, as it was considered too difficult for patients. For the SI condition, all four squares would turn green, and the subject had to form his/her own sequence of button-presses. As a feedback, the buttons pressed made the green squares turn yellow immediately, after which the next button was ready to be selected. Pressing the same button twice in a row was considered to be an error, indicated by the equivalent square turning red. We also asked the participants to refrain from using common sequences, such as 1-2-3-4 and 4-3-2-1, or repeating sequences, such as 4-2-3-1-4-2-3-1... Participants' performance was monitored to insure they kept their movement sequences as random as possible. During the ET condition, the subject had to follow the sequence as the blue squares alternately turned green. During the control condition, one square switched from blue to green, indicating that the corresponding button should be pressed. All the conditions with left and right hands were performed in every run of the fMRI scans, and the task included a total of 20 button presses per condition. For detailed task information, see (Francois-Brosseau et al., 2009; Martinu et al., 2012). The data during SI were used for this study.

\section{BEHAVIOR MEASUREMENT}

Individual averages of the RT during each task (SI, ET, and CTL) were calculated. The RT was defined as the time between the beginning of a button press to the beginning of the following button press. Data for the left and right hands were combined for the purpose of the present study. The averaged RT during SI movements was used as an index of performance level of subjects' behavior.

\section{fMRI SCANNING}

Subjects were scanned at the Unité de Neuroimagerie Fonctionnelle (UNF) of the Center de Recherche de l'Institut Universitaire de Gériatrie de Montréal (CRIUGM) using a 3T TIM Siemens Magnetom MRI scanner. Each scanning session began with a high- resolution, T1-weighted, three-dimensional volume acquisition for anatomical localization $\left(1 \mathrm{~mm}^{3}\right.$ voxel size), followed by four sets of echoplanar $\mathrm{T} 2{ }^{*}$-weighted image acquisitions with blood oxygenation level-dependent (BOLD) contrast (echo time, $30 \mathrm{~ms}$; flip angle, $90^{\circ}$ ). Each run consisted of 146 frames of 43 slices (matrix size, $128 \times 128$ pixels; voxel size, $3.34 \times 3.34 \times 3 \mathrm{~mm}^{3}$, TR: $3.5 \mathrm{~s}$ ). The number of total volume for each subject was 584 .

\section{fMRI PREPROCESSING}

We applied the NIAK preprocessing pipeline on fMRI data sets (Bellec et al., 2012). First, slice timing correction was performed with spline interpolation. After motion correction, slow time drift was removed from the BOLD time series with a high-pass filter of $0.015 \mathrm{~Hz}$. Images were then transformed into ICBM 152 space, and spatially blurred with a $4 \mathrm{~mm}$ full width half-maximum isotropic Gaussian kernel.

\section{REGIONS OF INTEREST (ROI) AND TIME SERIES EXTRACTION}

We generated task-related networks on specific tasks by selecting 62 regions of interest (ROI) as nodes. Fifty-eight ROIs were taken from the activation maps acquired by comparison between events (Martinu et al., 2012) in the HV with a threshold of $t=3.5$. They included 44 ROIs generated from the SI minus CTL contrast for left and right-hand movements combined, and 14 ROIs generated from the comparison between the right and left hands in the CTL condition. These ROIs included three of the putamen and three of the thalamus. We also added four ROIs, which were located in the caudate nucleus and the VS, bilaterally. All the ROIs are 
shown in the Table 1, with additional information for activation pattern. In graph theory, these ROIs are considered as nodes of the brain network, and the connections between every two ROIs are considered as edges. The ROIs consisted of small cubes (total of 27 voxels) centered on each peak in ICBM 152 space. Time series for the entire task duration ( $34 \mathrm{~min}, 584$ volumes) were extracted for each ROI for each subject and session.

\section{NORMALIZING AND DIVIDING TIME SERIES}

Each time series of single runs from the 62 ROIs were globally normalized using the average and standard deviation of all the extracted BOLD intensities (146 volumes $\times 62$ ROIs). The normalized time series were then divided into three, according to the task period (SI, ET, and CTL). Because of the hemodynamic delay inherent in BOLD signal after stimuli (Buxton and Frank, 1997), the first $10 \mathrm{~s}$ of each task period were removed. Finally, all the processed time series of the 4 runs during the SI were combined, individually, on which the network analysis was conducted.

\section{AVERAGED BOLD SIGNALS THROUGH TIME SERIES, AND ACTIVATION OF SI-CTL IN SUBCORTICAL REGIONS}

The normalized time series of 10 subcortical ROIs (caudate, putamen, VS, and the thalamus) during the SI were averaged for each subject. This measurement was considered as an index of regional activity during the tasks. For the PD group, from the activation maps acquired by comparison of SI - CTL (Martinu et al., 2012), individual $t$-values of brain activity was calculated. In the previous study, activation in the striatum and thalamus was reduced in the PD patients (Martinu et al., 2012). Therefore, the individual $t$-value in the subcortical ROIs was considered as an index of capacity/incapacity of the dopaminergic function in the PD patients.

\section{NETWORK ANALYSIS \\ Small-world property}

Using each individual data set of the normalized BOLD signal time course, Pearson's correlation coefficients was calculated between each pair the 62 ROIs, resulting in a symmetric $62 \times 62$ correlation matrix. Applying the cost-threshold approach on the $62 \times 62$ correlation matrix, we first checked the cost, which indicated small-world network property, showing global efficiency is less than random network but greater than a regular lattice network, and local efficiency is more than random network (Achard et al., 2006; Achard and Bullmore, 2007; Skidmore et al., 2011; Carbonell et al., 2014), in the HV and PD patients, respectively. Cost efficiency (global efficiency - cost) was calculated to see the economical cost, while maximizing the cost efficiency during the task, the HV and PD patients, respectively.

\section{Network structure-pattern of brain network in HV and PD}

The change of the connectivity based on cost was investigated by visual inspection. Based on the results of small-world property, cost efficiency, and visual inspection, we selected the cost of 0.28 to determine the individual networks for the SI task, in the $\mathrm{HV}$ and $\mathrm{PD}$, respectively. The edges included in more than half for each group were considered for the further analysis.
Table 1 | Regions of interest in the brain (in ICBM 152 space).

\begin{tabular}{lccc}
\hline ROI & $\boldsymbol{x}$ & $\boldsymbol{y}$ & $\boldsymbol{z}$ \\
\hline SI > ET, SI > CON & & & \\
SMA1 & -4 & -2 & 68 \\
PreSMA1 & 6 & 16 & 46 \\
VLPFC1 & -50 & 14 & 4 \\
VLPFC2 & 44 & 14 & 2 \\
VLPFC3 & 56 & 40 & 0 \\
DLPFC1 & 34 & 46 & 36 \\
APFC & 40 & 18 & 2 \\
INS1 & -32 & 20 & 4 \\
INS2 & 30 & -18 & 6 \\
Thal1 & -14 & -16 & 6 \\
Thal2 & 14 & -54 & 8 \\
LOA1 & 50 & & -6 \\
SI $>$ ET, SI $>$ CON, ET $>$ CON & -2 & 62 \\
PMd1 & -20 & -4 & 58 \\
PMd2 & 24 & -44 & 50 \\
IPL1 & 36 & 2 & 4 \\
Put1 & -24 & 4 & 8 \\
Put2 & 24 & &
\end{tabular}

\section{$\mathrm{SI}>\mathrm{CON}, \mathrm{ET}>\mathrm{CON}$}

PreSMA2

PreSMA3

PMd3

$\mathrm{PMd} 4$

IPL2

IPL3

IPL4

DLPFC2

DLPFC3

IPL5

IPL6

IPL7

SOG1

SOG2

SOG3

SOG4

MOG1

MOG2

MOG3

LG1

LG2

LG3

LG4

$\mathrm{FG} 1$

FG2

LOA2

Cereb1

MTR LEFT VS. RIGHT

M1-1

Cereb2

Cereb3

Cereb4

2

36

2 48

\section{8}

46 2 6 2 
Table 1 | Continued

\begin{tabular}{lccc}
\hline ROI & $\boldsymbol{x}$ & $\boldsymbol{y}$ & $\boldsymbol{z}$ \\
\hline Cereb5 & 28 & -66 & -52 \\
M1-2 & 40 & -22 & 54 \\
SMA2 & -6 & -20 & 50 \\
SMA3 & 8 & -24 & 50 \\
InsPost1 & -36 & -32 & 22 \\
InsPost2 & 42 & -22 & 20 \\
Cereb6 & -18 & -58 & -20 \\
Cereb7 & 20 & -52 & -20 \\
Thal3 & 16 & -20 & 2 \\
PutPost & 32 & -12 & 2 \\
ETC & & & 12 \\
Caudate1 & -14 & 12 & 12 \\
Caudate2 & 14 & 12 & -6 \\
VS1 & -10 & 14 & -6 \\
VS2 & 10 & 14 &
\end{tabular}

Abbreviations: SMA, supplemental motor area; PreSMA, pre-supplemental motor area; VLPFC, ventrolateral prefrontal cortex; APFC, anterior prefrontal cortex; INS, insula; Thal, thalamus; LOA, lateral occipital area; PMd, dorsal premotor cortex; IPL, intreaparietal lobe; DLPFC, dorsolateral prefrontal cortex; SOG, superior occipital gyrus; MOG, middle occipital gyrus; LG, lingual gyrus; FG, fusiform gyrus; M1, primary motor area; Cereb, cerebellum; Put, putamen; PutPost, posterior putamen; InsPost, posterior part of Insula; VS, ventral striatum.

\section{Group comparison between HV and PD}

To investigate group differences in overall connectivity, strength of the connectivity of all linkages (edges) during the SI was compared between groups. For this, Fischer's $\mathrm{Z}$ transformation was applied to each individual correlation matrix. Then, intergroup comparisons with $t$-test between the $\mathrm{HV}$ and PD groups were performed. Significance was set at $p<0.05$ (uncorrected). Combined with the results of the correlation analysis mentioned below, we explored the edges affected by the disease.

\section{Correlation analysis between correlation ratio of edges and activation of subcortical regions in $\mathrm{HV}$ and $P D$}

We performed a correlation analysis between the correlation ratio after Fisher's transformation of the edges above, and the activity in the subcortical regions, with SI condition. This analysis was done, in the HV and PD, respectively. The average BOLD signal through time series were used for the index of subcortical activity. In PD, t-activation calculated by SI - CTL conditions was also used for the analysis, as well as the index of incapacity of the dopaminergic function in PD patients. We reported the results with the significant threshold set at $p<0.05$ (uncorrected).

\section{Relationship between correlation ratio with subcortical connections and task performance in HV and PD}

Based on our previous study (Nagano-Saito et al., 2008), we assumed that stronger connectivity between the DLPFC and the striatum would correspond with the faster RT. We were also interested in whether any other correlation between other nodes and the subcortical regions, and weakened in PD, could explain the RT. We therefore calculated individual correlation ratio between these 32 regions and the striatum and the thalamus, then performed a correlation between the correlation ratio after Fisher's transformation and the RT(SI) in the HV and PD patients, respectively. The significant threshold was set at $p<0.05$ (uncorrected, for DLPFC, and FDR-corrected for others).

\section{Difference of path length from the primary motor area between groups}

The path length is the number of edges that the path uses. We assumed that the path length from primary motor area (M1) to the striatum, mPFC, and associative cortex is longer in $\mathrm{PD}$ patients compared with HV. To confirm this, we calculated the shortest path length from the left and right primary motor area to the other nodes, individually, and compared HV and PD. Wilcoxon rank sum test was used. We reported the results with the significant threshold set at $p<0.05$ (uncorrected).

\section{RESULTS}

\section{BEHAVIOR}

RT for the SI, ET, and CTL were 790.6 $\pm 208.3,1045.5 \pm$ $172.8,826.6 \pm 174.4 \mathrm{~ms}$ respectively in $\mathrm{HV}$, and $888.67 \pm 161.4$, $1152.6 \pm 208.7,733.2 \pm 112.3 \mathrm{~ms}$ respectively in PD patients. A mixed-design repeated measures ANOVA (task $\times$ group) showed a significant main effect of the task $(F=44.956$; $p<$ $0.001)$, but not group $(F=0.108 ; p=0.745)$. There was also a significant interaction of task $\times$ group $(F=5.178 ; p=0.016)$, showing that the RT for SI was shorter than for CTL in HV, and RT for CTL was shorter than SI in PD patients.

\section{NETWORK ANALYSIS}

\section{Small-world network property}

All the networks in the HV and PD showed small-world network property in the range of the cost of [0.045-0.460], and of [0.080-0.485], respectively, with less global efficiency and more local efficiency, compared to random networks, and more global efficiency compared to lattice networks (Figure 1). After smoothing the group averaged data using matlab toolbox (preserving the shape option), we obtained the peaks of the cost efficiency at the cost of 0.271 and 0.284 , for the HV and PD groups, respectively, and we selected the cost of 0.28 for further analyses.

\section{Network structure-pattern of brain network in HV and PD}

Cost-dependent patterns of brain network during SI are shown in the Figure 2. In both groups, connectivity in the dorsal and ventral regions of the brain was observed above the cost of 0.20 . With the individual cost of 0.28 , the mean correlation threshold (the minimal correlation ratio) was $0.261 \pm 0.0064$ in $\mathrm{HV}$ and 0.245 \pm 0.0582 in PD group, and the total connectivity with our criteria was $12.6 \%$ in $\mathrm{HV}$, and $10.9 \%$ in the PD group. The connectivity with striatum and the thalamus (excluding inter-connections) were confined to the extrastriate visual cortex and the VLPFC and insula. All the connectivities in the HV and PD, respectively, with our criteria are shown in the Complementary Data (C-Table 1). 

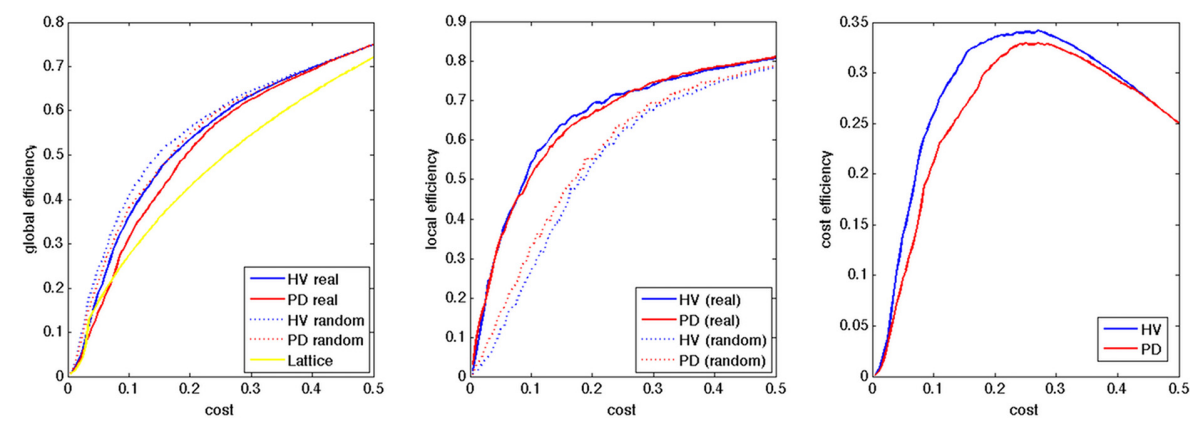

FIGURE 1 | Group average of global efficiency, local efficiency, and cost efficiency in HV and PD. The X axis indicates the wiring cost.

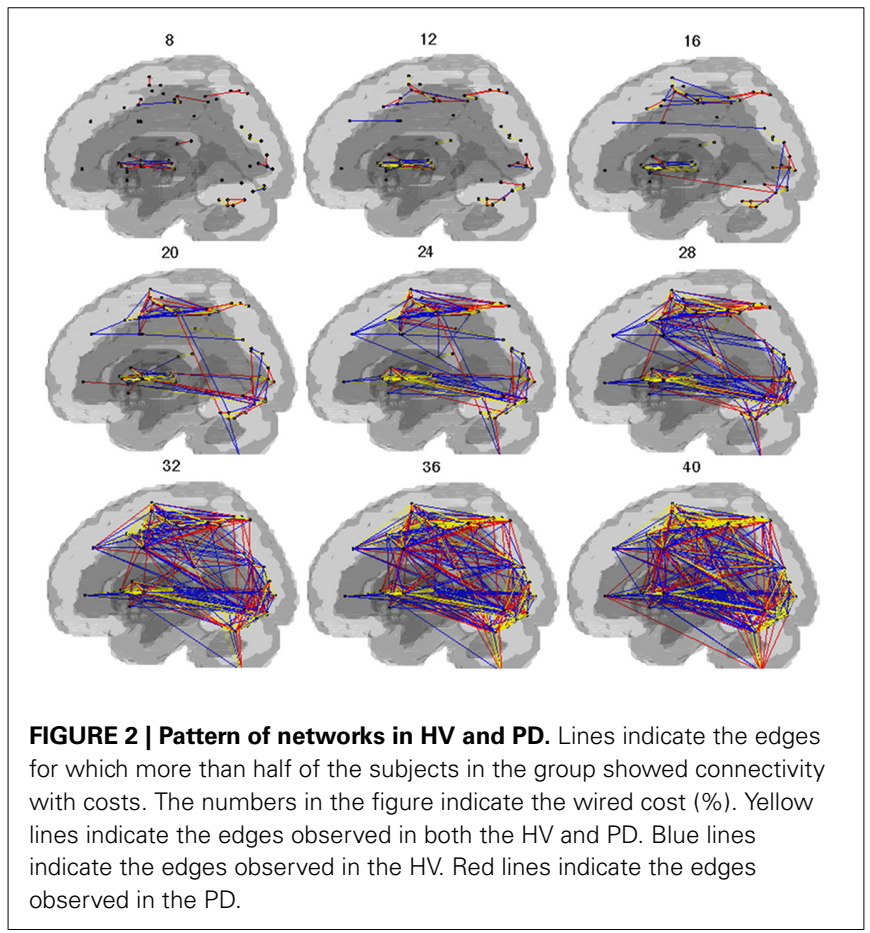

\section{Group comparison between HV and PD}

All results are shown in the Tables 2, 3. The PD patients show weakened connectivity between the MPFC and other cortical areas, including the primary motor area (M1), the extrastriate visual cortex and the associative cortex. Simultaneously, however, PD patients display a few regions of increased connectivity with the mPFC. In the PD patients, the striatum and the thalamus have reduced connectivity with the extrastriate visual cortex, and the connectivity between the VLPFC and the striatum, between the M1 and the posterior insula, as well as with the cerebellum (Cereb) for the left and right sides, respectively, was weakened. Inter connectivity between the left and right DLPFC and within the subcortical regions were also reduced in the PD patients. However, the connectivity between the bilateral VS and intra extrastriate visual cortex was stronger in the PD.
Table 2 | Differences in edge strength connectivity between HV and PD during the finger movement tasks (HV > PD).

\begin{tabular}{|c|c|c|c|c|}
\hline \multicolumn{2}{|c|}{ Edges (HV > PD) (OFF) } & \multirow[t]{2}{*}{$p$-value } & \multicolumn{2}{|c|}{ Subcortical activity } \\
\hline & & & HV & PD \\
\hline PreSMA1 & DLPFC1 & 0.0463 & * & * \\
\hline PreSMA1 & IPL2 & 0.0232 & * & * \\
\hline PreSMA1 & SMA3 & 0.0003 & $*$ & * \\
\hline PreSMA1 & InsPost1 & 0.0023 & * & * \\
\hline PreSMA1 & SOG4 & 0.0342 & & * \\
\hline PreSMA1 & SMA2 & 0.0434 & * & \\
\hline PreSMA1 & SOG1 & 0.0263 & * & * \\
\hline PreSMA2 & M1-1 & 0.0094 & & * \\
\hline PreSMA2 & SMA2 & 0.0392 & * & * \\
\hline PreSMA3 & PMd2 & 0.0246 & & * \\
\hline SMA1 & M1-1 & 0.022 & & * \\
\hline SMA1 & IPL5 & 0.0444 & & * \\
\hline SMA2 & DLPFC2 & 0.0415 & & * \\
\hline DLPFC1 & DLPFC2 & 0.0249 & * & \\
\hline DLPFC3 & SOG1 & 0.002 & * & * \\
\hline DLPFC3 & SOG3 & 0.0331 & & * \\
\hline M1-1 & InsPost1 & 0.0319 & & \\
\hline M1-2 & Cereb6 & 0.0489 & & \\
\hline VLPFC3 & Put1 & 0.0362 & & * \\
\hline Put2 & Thal1 & 0.0236 & * & \\
\hline Put2 & Thal2 & 0.0131 & * & * \\
\hline PutPost & VS1 & 0.0136 & & * \\
\hline VS1 & LG3 & 0.0022 & * & \\
\hline Thal1 & LG2 & 0.0079 & * & * \\
\hline Thal1 & FG1 & 0.0147 & & \\
\hline Thal3 & MOG3 & 0.0226 & * & \\
\hline Thal3 & $\mathrm{FG} 1$ & 0.0054 & & \\
\hline
\end{tabular}

Edges whose connectivity strength was positively correlated to subcortical (striatum and/or thalamus) activation are marked by an asterisk.

\section{Correlation analysis between correlation ratio of edges and activation of subcortical regions in $\mathrm{HV}$ and PD}

Fourteen out of the 27 edges for which connectivity strength was weakened in PD patients were correlated with the subcortical 
Table 3 | Difference in edge strength connectivity between HV and PD during the finger movement tasks (PD > HV).

\begin{tabular}{lll}
\hline \multicolumn{2}{c}{ Edges (HV < PD) } & p-value \\
\hline PreSMA3 & LG4 & 0.0489 \\
SMA2 & Cereb7 & 0.0088 \\
SMA3 & IPL1 & 0.0388 \\
VS1 & VS2 & 0.0077 \\
MOG1 & LG1 & 0.0229 \\
MOG1 & LG2 & 0.0448 \\
\hline
\end{tabular}

activation during the SI task in HV (Table 4, Figure 3). All the edges that are significantly correlated with subcortical regions are marked with an asterisk in the Table 2. Eighteen of those connection strengths were correlated with the $t$-value of the SI - CTL contrast in the PD patients (Tables 2, 4, Figure 3). Although nine edges overlapped between $\mathrm{HV}$ and $\mathrm{PD}$, the correlation patterns were different between the groups. In $\mathrm{HV}$, the correlation between the correlation ratio of the edges and the striatal activation was positive, whereas the correlation between the correlation ratio of the edges and thalamic activation was negative (Table 4). In contrast, in PD, the correlation was positive for the striatum as well as the thalamus. Of note, none of the connectivity was significantly correlated with RT.

\section{Relationship between correlation ratio of the cortico-subcortical connection and task performance in HV and PD}

The connectivity strength between the right DLPFC (DLPFC1 and DLPFC3, in the Table 1) and the striatum was negatively correlated with RT $(r=-0.557 ; p=0.038, r=-0.596$; $p=0.245 ; r=-0.553, p=0.040$, respectively) (Figure 4). The connectivity strength between right VLPFC (VLPFC3, in the Table 1) and the striatum was strongly positively correlated with RT $(r=0.857$; uncorrected $p=0.00006$, corrected $p=0.0105)$.

\section{Difference of path length from the primary motor area and other nodes in HV and PD}

The path length from the left M1 to the pre-SMA and SMA, left and right DLPFC, and the right posterior putamen, was shorter in the HV compared with PD. The path length from the right $\mathrm{M} 1$ to the right insula, right thalamus, right dorsal premotor cortex (PMd), and the left lingual gyrus (LG) was longer in the HV. Results are shown in the Table 5. The mean lengths form the M1-1 to these brain regions as a function of cost are shown in the Complementary data (C-Figure 1).

\section{DISCUSSION}

The BG, modulated by dopaminergic projections (Schultz, 1998; Seamans and Yang, 2004; Joshua et al., 2009; Howe et al., 2013), are thought to play a crucial role in the integration of information from multiple sources in order to make a decision and perform an optimized action (Bar-Gad et al., 2003; Bogacz and Gurney, 2007). The dopaminergic neurons might be involved in the formation of an ideal network combining the cognitive and motor networks in the brain for conducting a series of tasks. We used
Table 4 | Correlation between edge strength and subcortical activity.

Edges (HV > PD) Subcortical regions $r$ (normalized) $p$-value

\begin{tabular}{lllrl}
\hline HV (SUBCORITICAL ACTIVATION) & & \\
DLPFC1 & DLPFC2 & PutPost & 0.619 & 0.018 \\
DLPFC3 & SOG1 & VS2 & 0.568 & 0.034 \\
PreSMA1 & DLPFC1 & VS1 & 0.563 & 0.036 \\
PreSMA1 & DLPFC1 & VS2 & 0.587 & 0.027 \\
PreSMA1 & DLPFC1 & Thal1 & -0.694 & 0.006 \\
PreSMA1 & InsPost1 & Put2 & 0.548 & 0.043 \\
PreSMA1 & IPL2 & Caudate1 & 0.645 & 0.013 \\
PreSMA1 & IPL2 & Caudate2 & 0.639 & 0.014 \\
PreSMA1 & IPL2 & Thal1 & -0.655 & 0.011 \\
PreSMA1 & IPL2 & Thal2 & -0.601 & 0.023 \\
PreSMA1 & SMA2 & Caudate2 & 0.655 & 0.011 \\
PreSMA1 & SMA2 & VS2 & 0.639 & 0.014 \\
PreSMA1 & SMA2 & Thal1 & -0.750 & 0.002 \\
PreSMA1 & SMA2 & Thal2 & -0.721 & 0.004 \\
PreSMA1 & SMA3 & VS2 & 0.753 & 0.002 \\
PreSMA1 & SMA3 & Thal2 & -0.628 & 0.016 \\
PreSMA1 & SOG1 & Thal1 & -0.628 & 0.016 \\
PreSMA2 & SMA2 & Thal1 & -0.576 & 0.031 \\
PreSMA2 & SMA2 & Thal2 & -0.637 & 0.014 \\
Thal1 & Put2 & PutPost & 0.690 & 0.006 \\
Thal1 & Put2 & Thal1 & -0.570 & 0.033 \\
Thal1 & LG2 & PutPost & 0.569 & 0.034 \\
Thal2 & Put2 & PutPost & 0.725 & 0.003 \\
LG3 & VS1 & PutPost & 0.581 & 0.029 \\
PMOG & Tha3 & PutPost & 0.544 & 0.044
\end{tabular}

$\begin{array}{llll}\text { MOG3 Thal3 PutPost } & 0.544 & 0.044\end{array}$

\section{PD (SUBCORITICAL ACTIVATION)}

$\begin{array}{lllll}\text { PreSMA2 } & \text { M1-1 } & \text { Thal2 } & 0.581 & 0.048 \\ \text { DLPFC3 } & \text { SOG3 } & \text { Put1 } & 0.624 & 0.030\end{array}$

$\begin{array}{lllll}\text { PD (SI-CTL) (t-VALUE) } & & \\ \text { DLPFC1 } & \text { PreSMA1 } & \text { Put2 } & 0.698 & 0.012\end{array}$

$\begin{array}{llll}\text { DLPFC1 PreSMA1 Thal1 } & 0.583 & 0.047\end{array}$

$\begin{array}{llll}\text { DLPFC1 SMA2 VS1 } & 0.632 & 0.027\end{array}$

$\begin{array}{llll}\text { DLPFC2 SMA2 Thal1 } & 0.677 & 0.015\end{array}$

$\begin{array}{llll}\text { DLPFC2 SMA2 Thal2 } & 0.656 & 0.021\end{array}$

$\begin{array}{llll}\text { DLPFC3 SOG1 } & \text { Caudate2 } & 0.578 & 0.049\end{array}$

$\begin{array}{llll}\text { PreSMA1 InsPost1 VS2 } & 0.658 & 0.020\end{array}$

$\begin{array}{lllll}\text { PreSMA1 IPL2 } & \text { Put2 } & 0.675 & 0.016\end{array}$

$\begin{array}{lllll}\text { PreSMA1 IPL2 } & \text { Thal1 } & 0.664 & 0.019\end{array}$

$\begin{array}{llll}\text { PreSMA1 SMA3 } & \text { Caudate1 } & 0.648 & 0.023\end{array}$

$\begin{array}{llll}\text { PreSMA1 SOG1 VS1 } & 0.645 & 0.024\end{array}$

$\begin{array}{llll}\text { PreSMA1 SOG4 Put2 } & 0.616 & 0.033\end{array}$

$\begin{array}{llll}\text { PreSMA1 SOG4 PutPost } & 0.584 & 0.046\end{array}$

$\begin{array}{llll}\text { PreSMA1 SOG4 } & \text { Thal1 } & 0.761 & 0.004\end{array}$

$\begin{array}{llll}\text { PreSMA1 SOG4 } & \text { Thal2 } & 0.686 & 0.014\end{array}$

$\begin{array}{llll}\text { PreSMA2 M1-1 PutPost } & 0.676 & 0.016\end{array}$

$\begin{array}{llll}\text { PreSMA2 SMA2 PutPost } & 0.674 & 0.016\end{array}$

$\begin{array}{llll}\text { PreSMA2 SMA2 } & \text { Caudate1 } & 0.717 & 0.009\end{array}$

$\begin{array}{llll}\text { PreSMA2 SMA2 Thal1 } & 0.672 & 0.017\end{array}$

$\begin{array}{llll}\text { PreSMA2 SMA2 Thal2 } & 0.741 & 0.006\end{array}$

$\begin{array}{llll}\text { PreSMA3 PMd2 VS1 } & 0.691 & 0.013\end{array}$

$\begin{array}{llll}\text { PreSMA3 PMd2 VS2 } & 0.607 & 0.036\end{array}$

(Continued) 
Table 4 | Continued

\begin{tabular}{|c|c|c|c|c|}
\hline \multicolumn{2}{|c|}{ Edges $(H V$ > PD) } & \multirow{2}{*}{$\begin{array}{l}\text { Subcortical regions } \\
\text { VS1 }\end{array}$} & \multirow{2}{*}{$\begin{array}{c}\boldsymbol{r} \text { (normalized) } \\
0.696\end{array}$} & \multirow{2}{*}{$\begin{array}{r}\boldsymbol{p} \text {-value } \\
0.012\end{array}$} \\
\hline SMA1 & IPL5 & & & \\
\hline SMA1 & M1-1 & Thal2 & 0.750 & 0.005 \\
\hline Put1 & VLPFC3 & Caudate1 & 0.629 & 0.029 \\
\hline Put1 & VLPFC3 & VS1 & 0.590 & 0.043 \\
\hline Put1 & VLPFC3 & VS2 & 0.586 & 0.045 \\
\hline Put2 & Thal2 & Caudate1 & 0.689 & 0.013 \\
\hline PutPost & VS1 & Put2 & 0.700 & 0.011 \\
\hline PutPost & VS1 & VS2 & -0.752 & 0.005 \\
\hline Thal1 & LG2 & Put2 & 0.629 & 0.029 \\
\hline Thal1 & LG2 & Thal1 & 0.619 & 0.032 \\
\hline
\end{tabular}

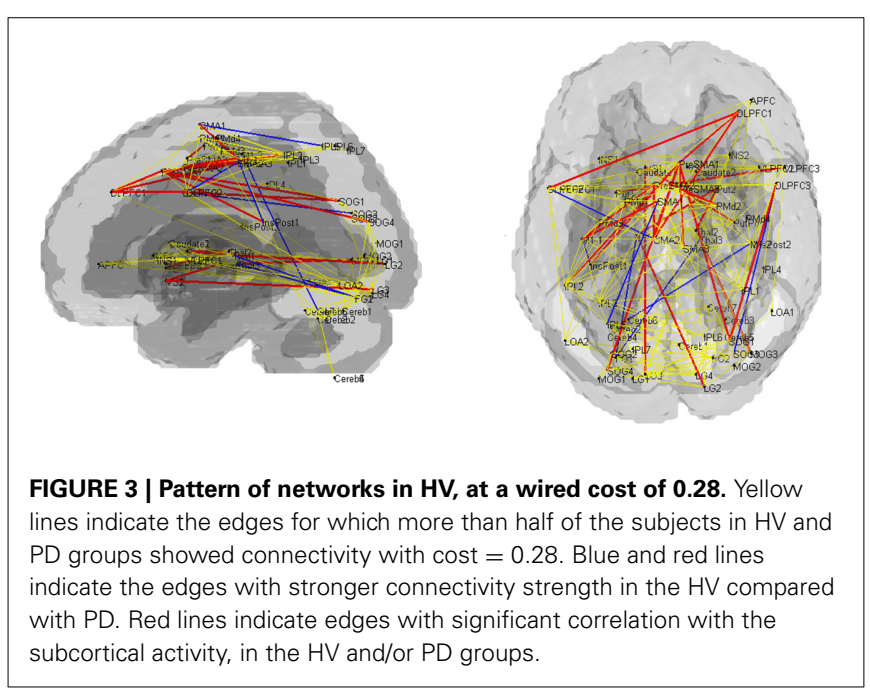

a graph theory approach on SI finger movements during fMRI acquisition in order to investigate this function in $\mathrm{HV}$ and PD patients.

\section{GRAPH-THEORY APPROACH}

We used the graph-theory approach while considering wiring cost to select edges relevant for the SI finger movement task used. This could improve the detection of pivotal edges affected by the disease. The peak of the cost efficiency was located at the cost of 0.271 and 0.284 in the HV and PD groups, respectively. Based on a hypothesis that the brains operate optimally with the maximum cost efficiency, maximizing information transfer (Sporns et al., 2000; Achard and Bullmore, 2007), we selected the cost of 0.28 to determine individual networks. Then, the edges included in more than half of the subjects for each group were considered, considering them as a common network for the task. Costs of 0.20 and 0.36 were also calculated, and the results were comparable to the results with a cost of 0.28 (data not shown). Additionally, the results using correlation threshold were also equivalent (patterns of networks with this method are shown in Complementary Data, C-Figure 2), similar to our previous study showing relatively preserved results with the two thresholding methods (Carbonell et al., 2014).
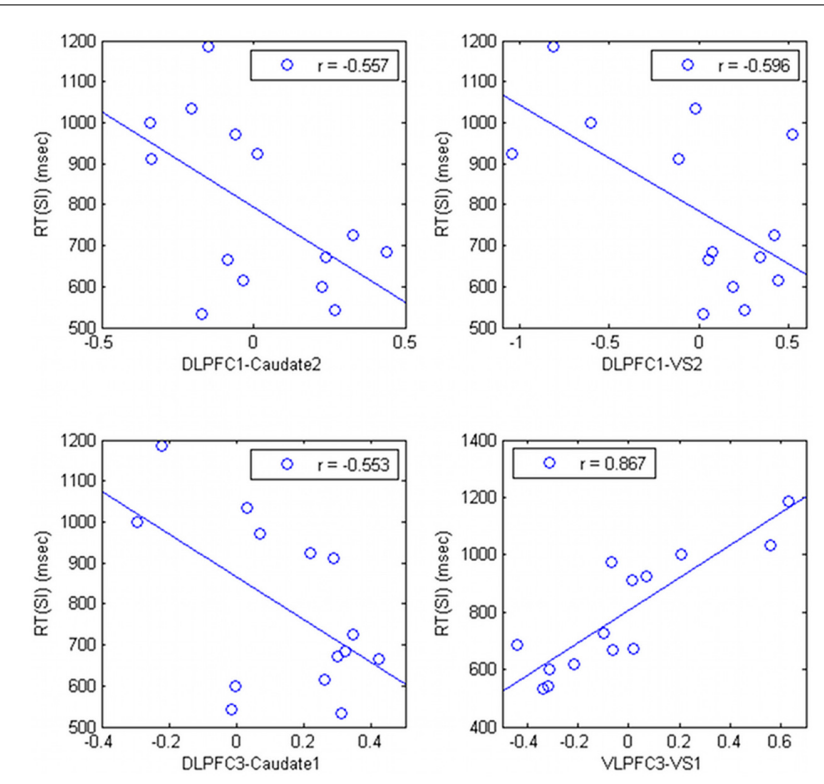

FIGURE 4 | Relationship between correlation ratio of the cortico-subcortical connections and task performance in HV.

Table 5 | Difference in path length between the primary motor area and other nodes.

\begin{tabular}{llccc}
\hline \multirow{2}{*}{ Nodes } & \multicolumn{2}{c}{ Path length $($ mean \pm SD) } & \multirow{2}{*}{$\boldsymbol{p}$-value } \\
\cline { 3 - 4 } & & HV & PD & \\
\hline \multirow{2}{*}{ M1-1 } & SMA1 & $1.429 \pm 0.646$ & $2.083 \pm 0.669$ & 0.019 \\
& PreSMA1 & $1.643 \pm 0.633$ & $2.250 \pm 0.622$ & 0.027 \\
& PreSMA2 & $1.429 \pm 0.646$ & $2.083 \pm 0.669$ & 0.019 \\
& DLPFC1 & $1.643 \pm 0.745$ & $2.333 \pm 0.778$ & 0.033 \\
& DLPFC2 & $1.571 \pm 0.646$ & $2.250 \pm 0.622$ & 0.016 \\
& PutPost & $2.000 \pm 0.392$ & $2.417 \pm 0.669$ & 0.049 \\
\hline \multirow{2}{*}{ M1-2 } & INS2 & $2.357 \pm 0.497$ & $1.833 \pm 0.577$ & 0.028 \\
& Thal2 & $2.214 \pm 0.579$ & $1.667 \pm 0.651$ & 0.037 \\
& PMd3 & $1.929 \pm 0.475$ & $1.417 \pm 0.515$ & 0.019 \\
& LG2 & $2.714 \pm 0.469$ & $2.167 \pm 0.718$ & 0.040 \\
\hline
\end{tabular}

\section{CORTICO-MIDLINE CONNECTIVITY IS MODULATED BY BG ACTIVITY}

The main finding of the present study is that connectivity between the MPFC and the motor cortex, extrastriate cortex, and the associative cortex, is weakened in comparison to HV. Furthermore, the strength of the cortico-midline connections was correlated to striatal and thalamic activity. This is in accordance with a previous study that showed a decrease in functional connectivity between the pre-SMA and M1, PMC, IPL, and the cerebellum in PD patients during a SI task (Wu et al., 2011). Our results additionally demonstrate that (1) the lowered connectivity between the MPFC and other cortices remain prominent within the whole brain network, and (2) that the activation in the striatum (the caudate, putamen, and VS) and the thalamus would be involved in supporting this connectivity. 
We observed weakened connections between the pre-SMA and M1 in PD patients, and this was accompanied by a reduction of activity in the right posterior putamen, the motor nucleus of the BG (Alexander et al., 1986; Albin et al., 1989). The pre-SMA has anatomical connections with prefrontal areas, without direct connections to the primary motor area (Picard and Strick, 2001). In addition, the pre-SMA is connected to the caudate nucleus, the associative nucleus of the BG, the rostral part of putamen and the globus pallidus (Takada et al., 1998; Lehericy et al., 2004; Akkal et al., 2007). Functionally, the pre-SMA is in a position to influence the motor cortex during action selection (Mars et al., 2009). There may therefore be functional connectivity between the preSMA and M1 mediated by the BG system. Interestingly, the mPFC (pre-SMA and SMA) is one of the most frequently reported regions in connectivity analyses (Toro et al., 2008). Moreover, the mPFC, along with other midline structures, is considered to be a structural hub, with dense connectivity to the rest of the brain (Hagmann et al., 2008). A stronger connectivity between the preSMA and M1, then, would result in shortening in the path length between M1 and the other cortical regions. In accordance with this hypothesis, we found shorter path lengths between the M1 and the pre-SMA, SMA, putamen, and DLPFC in HV compared with PD patients.

Although striatal and thalamic activity was decreased in PD patients compared with HV (Martinu et al., 2012), the strength of cortico-midline connectivity was positively correlated with activity in the striatum, but negatively correlated with activity in the thalamus in the HV. In contrast, in the PD group, correlations with the cortico-midline connectivity were positive for the striatum as well as the thalamus. The striatum and the thalamus are tightly inter-connected through the cortico-BG-thalamocortical loops and the brainstem-thalamo-BG-brainstem loops (Alexander et al., 1986; Albin et al., 1989; McHaffie et al., 2005). Within these circuits, the indirect pathway of the corticoBG-thalamo-cortical loops exerts opposing effects on the striatum and the thalamus (Alexander et al., 1986; Albin et al., 1989; McHaffie et al., 2005). Therefore, the opposite correlation observed in HV may indicate the involvement of the indirect pathway in the control of cortico-midline connections. Recent animal studies have shown that the direct and indirect pathways are concurrently activated during operant tasks, suggesting that the indirect pathway is positively involved in decision making of actions, rather than simply suppressing unselected actions (Cui et al., 2013; Isomura et al., 2013). Indeed, our observations are in accordance with these recent views.

\section{CONNECTIVITY BETWEEN THE EXTRASTRIATE VISUAL CORTEX AND SUBCORTICAL REGIONS}

Another important observation in the present study was the decrease in connectivity between the extrastriate visual cortex and the subcortical regions (thalamus and striatum), between the striatum and the thalamus, as well as between the putamen and the VLPFC in the PD group. Our observation suggests that dopamine is involved in controlling the strength of the connectivity. The SI task was designed for internally generated movements. However, the subjects received a visual feedback for pressing a button. Therefore, the task also has a feature of visual-cue-guided goal-directed behavior, showing activation in the visual areas. The extrastriate visual cortex is interconnected with the thalamus at the level of the pulvinar (Jacobson and Marcus, 2007; Leh et al., 2008). In monkeys, this region is connected to the body and tail of the caudate nucleus (Yeterian and Pandya, 1995). The VLPFC is also strongly connected to the striatum, especially in the rostro-ventral part of the caudate nucleus (Leh et al., 2007). Also, the visual cortex also shows functional connectivity with the VLPFC/insula during a visualization task (Ebisch et al., 2013). Nevertheless, the thalamic and striatal regions observed in the present study included the motor components located in the ventrolateral/ventral-posterolateral thalamus and the putamen (Jacobson and Marcus, 2007). Our results suggest, then, a level of integration between subcortical regions involved in visual and motor processes.

\section{INTEGRATION OF INFORMATION BETWEEN THE MOTOR CORTEX AND THE PREFRONTAL CORTEX}

Connectivity strength between the superior occipital gyri and the DLPFC showed significant decreases in PD that correlated with subcortical activations. This is in line with a previous study that showed attention-induced fronto-posterior connectivity to be modulated by the BG (van Schouwenburg et al., 2010). The connectivity between the DLPFC and the striatum was not significant (Figure 3), but was negatively correlated to the RT in HV (Figure 4). These observations are in accordance with our previous study, where an increase in temporal connectivity between the DLPFC and the dorsal striatum was associated with shorter RT (Nagano-Saito et al., 2008). It has been suggested that the general role of the DLPFC is to establish a set of responses suitable for a given task (Nathaniel-James and Frith, 2002). Additionally, the DLPFC belongs to the fronto-parietal task control network, which is considered to play a role in the initiation of movement, and in trial-by-trial adaptive control (Dosenbach et al., 2007, 2008). In these respects, the connectivity between the DLPFC and the striatum may correspond to an information flow for trialby-trial adaptation supported by the large background of the fronto-parietal task control network.

The connectivity between the VLPFC and the striatum was also decreased in PD. This is in accordance with our previous study where we showed that temporal connectivity between the VLPFC and the striatum was reduced when dopamine was depleted (Nagano-Saito et al., 2008). However, the connectivity between the VLPFC and the striatum, as well as the connectivity between the extrastriate visual cortex and the subcortical regions were strong during the task, as opposed to the connectivity between the DLPFC and the striatum (Figure 2). Furthermore, the strength of the connectivity was positively correlated with the individual RT in the HV (Figure 4). We speculated that the stronger connectivity between the VLPFC and the subcortical regions be linked with an increase in visual information flow, which contributes to inhibiting behavioral responses (Sakagami et al., 2001). This may help cognitive function, but requires additional processing for action decision, possibly leading to a longer RT.

Considering the fact that the DLPFC and the VLPFC are endpoints of the ventral and dorsal visual pathways (Sakagami 
and Pan, 2007), our observation may imply that the subcortical regions play a role in connecting the two visual pathways. This is consistent with a speculation that the BG system would have an important role in the integration of the dorsal and ventral visual pathways' information for decision-making (Cisek and Kalaska, 2010).

The pre-SMA and VLPFC are directly inter-connected (Aron, 2007), and are considered to be a part of the cingulo-opecular task control network involved in set maintenance (Dosenbach et al., 2007, 2008; Power et al., 2011). Although the pre-SMA and VLPFC show synchronized activity during the resting state, we did not observe strong connectivity between them during the SI task (Figure 2), suggesting the two regions have distinct functions. Accordingly, a recent study showed differential modulation of functional connectivity between the VLPFC and the pre-SMA during a reasoning task (Ebisch et al., 2013). More specifically, the VLPFC showed an increase in functional coupling with visual cortices during a visualization task, whereas the pre-SMA displayed an increase in functional coupling with the anterior frontal cortex during an induction task (Ebisch et al., 2013). The pre-SMA and the VLPFC may function for cognitive tasks collaboratively. However, at least in specific tasks, the preSMA would allow the establishment of a shorter path between the motor cortex and the other cortical regions, whereas the VLPFC would allow the collection of visual information to the subcortical regions. The striatum and the thalamus, then, may further reinforce these functional connections to generate adequate decisions on resulting actions.

We speculated that mediation of cognitive and motor modules supported by these PFC and subcortical regions in the brain would be important for decision-making processes.

In humans, co-activation of the pre-SMA, VLPFC, DLPFC, along with the thalamus and the striatum has been repeatedly reported during cognitive tasks that require a task-set for visuallyguided goal-directed responses (Monchi et al., 2001; Huettel et al., 2004; Chang et al., 2007; Nagano-Saito et al., 2008), and even during our SI tasks (Martinu et al., 2012). Although the exact mechanism behind this integration is unclear, it is most likely mediated by the striato-nigro-striatal connections (Haber, 2003) and the cortico-BG-thalamo-cortical loops (McFarland and Haber, 2002; Calzavara et al., 2007; Draganski et al., 2008).

\section{CONCLUSION}

In the present study, we have applied a graph theory approach to fMRI data. Our results support the hypothesis that through dopaminergic neuro-modulation (Seamans and Yang, 2004; Joshua et al., 2009; Howe et al., 2013), the BG play an important role in the integration of multiple sources of information from functionally specific cortical areas in order to generate an adequate decision on a resulting action (Bar-Gad et al., 2003; Bogacz and Gurney, 2007). More specifically, the BG would integrate information dorsally from parieto-frontal areas, and ventrally from visual areas. Additionally, the BG would allow to shorten the path length between the mPFC and the motor cortex. In networking terms, then, mPFC, DLPFC, and VLPFC interact with the BG to create the adequate network that can combine visual, associative, and motor information to produce the decision on an upcoming action. A possible model for SI task is

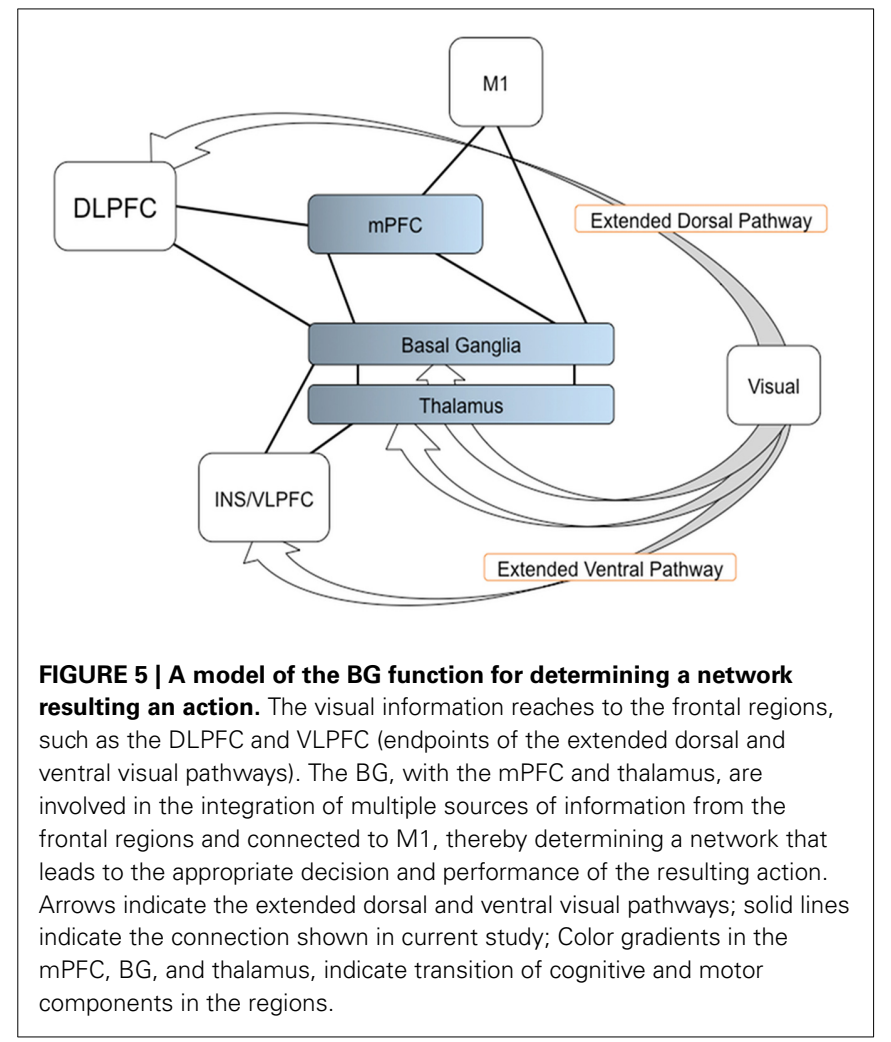

shown in Figure 5. Additional studies will be necessary in order to determine whether this effect is task-dependent.

\section{ACKNOWLEDGMENTS}

This work was supported by a Canadian Institutes of Health Research grant (MOP 126017), and a Parkinson Society Canada psychosocial grant to Oury Monchi. The authors would like to thank the staff at the Functional Neuroimaging Unit of the CRIUGM.

\section{SUPPLEMENTARY MATERIAL}

The Supplementary Material for this article can be found online at: http://www.frontiersin.org/journal/10.3389/fnins.2014. 00187/abstract

\section{REFERENCES}

Achard, S., and Bullmore, E. (2007). Efficiency and cost of economical brain functional networks. PLoS Comput. Biol. 3:e17. doi: 10.1371/journal.pcbi.0030017

Achard, S., Salvador, R., Whitcher, B., Suckling, J., and Bullmore, E. (2006). A resilient, low-frequency, small-world human brain functional network with highly connected association cortical hubs. J. Neurosci. 26, 63-72. doi: 10.1523/JNEUROSCI.3874-05.2006

Akkal, D., Dum, R. P., and Strick, P. L. (2007). Supplementary motor area and presupplementary motor area: targets of basal ganglia and cerebellar output. J. Neurosci. 27, 10659-10673. doi: 10.1523/JNEUROSCI.3134-07.2007

Albin, R. L., Young, A. B., and Penney, J. B. (1989). The functional anatomy of basal ganglia disorders. Trends Neurosci. 12, 366-375. doi: 10.1016/01662236(89)90074-X

Alexander, G. E., DeLong, M. R., and Strick, P. L. (1986). Parallel organization of functionally segregated circuits linking basal ganglia and cortex. Annu. Rev. Neurosci. 9, 357-381. doi: 10.1146/annurev.ne.09.030186.002041

Aron, A. R. (2007). The neural basis of inhibition in cognitive control. Neuroscientist 13, 214-228. doi: 10.1177/1073858407299288 
Baradaran, N., Tan, S. N., Liu, A., Ashoori, A., Palmer, S. J., Wang, Z. J., et al. (2013). Parkinson's disease rigidity: relation to brain connectivity and motor performance. Front. Neurol. 4:67. doi: 10.3389/fneur.2013.00067

Bar-Gad, I., Morris, G., and Bergman, H. (2003). Information processing, dimensionality reduction and reinforcement learning in the basal ganglia. Prog. Neurobiol. 71, 439-473. doi: 10.1016/j.pneurobio.2003.12.001

Bassett, D. S., and Bullmore, E. (2006). Small-world brain networks. Neuroscientist 12, 512-523. doi: 10.1177/1073858406293182

Bassett, D. S., Bullmore, E. T., Meyer-Lindenberg, A., Apud, J. A., Weinberger, D. R., and Coppola, R. (2009). Cognitive fitness of cost-efficient brain functional networks. Proc. Natl. Acad. Sci. U.S.A. 106, 11747-11752. doi: 10.1073/pnas.0903641106

Bassett, D. S., Wymbs, N. F., Porter, M. A., Mucha, P. J., Carlson, J. M., and Grafton, S. T. (2011). Dynamic reconfiguration of human brain networks during learning. Proc. Natl. Acad. Sci. U.S.A. 108, 7641-7646. doi: 10.1073/pnas.1018985108

Bellec, P., Lavoie-Courchesne, S., Dickinson, P., Lerch, J. P., Zijdenbos, A. P., and Evans, A. C. (2012). The pipeline system for Octave and Matlab (PSOM): a lightweight scripting framework and execution engine for scientific workflows. Front. Neuroinform. 6:7. doi: 10.3389/fninf.2012.00007

Biswal, B., Yetkin, F. Z., Haughton, V. M., and Hyde, J. S. (1995). Functional connectivity in the motor cortex of resting human brain using echo-planar MRI. Magn. Reson. Med. 34, 537-541. doi: 10.1002/mrm.1910340409

Bogacz, R., and Gurney, K. (2007). The basal ganglia and cortex implement optimal decision making between alternative actions. Neural Comput. 19, 442-477. doi: 10.1162/neco.2007.19.2.442

Bullmore, E., and Sporns, O. (2009). Complex brain networks: graph theoretical analysis of structural and functional systems. Nat. Rev. Neurosci. 10, 186-198. doi: $10.1038 / \mathrm{nrn} 2575$

Buxton, R. B., and Frank, L. R. (1997). A model for the coupling between cerebral blood flow and oxygen metabolism during neural stimulation. J. Cereb. Blood Flow Metab. 17, 64-72. doi: 10.1097/00004647-199701000-00009

Calzavara, R., Mailly, P., and Haber, S. N. (2007). Relationship between the corticostriatal terminals from areas 9 and 46, and those from area 8A, dorsal and rostral premotor cortex and area $24 \mathrm{c}$ : an anatomical substrate for cognition to action. Eur. J. Neurosci. 26, 2005-2024. doi: 10.1111/j.1460-9568.2007.05825.x

Cao, H., Xu, X., Zhao, Y., Long, D., and Zhang, M. (2011). Altered brain activation and connectivity in early Parkinson disease tactile perception. AJNR Am. J. Neuroradiol. 32, 1969-1974. doi: 10.3174/ajnr.A2672

Carbonell, F., Nagano-Saito, A., Leyton, M., Cisek, P., Benkelfat, C., He, Y., et al. (2014). Dopamine precursor depletion impairs structure and efficiency of resting state brain functional networks. Neuropharmacology 84, 90-100. doi: 10.1016/j.neuropharm.2013.12.021

Chang, C., Crottaz-Herbette, S., and Menon, V. (2007). Temporal dynamics of basal ganglia response and connectivity during verbal working memory. Neuroimage 34, 1253-1269. doi: 10.1016/j.neuroimage.2006.08.056

Cisek, P., and Kalaska, J. F. (2010). Neural mechanisms for interacting with a world full of action choices. Annu. Rev. Neurosci. 33, 269-298. doi: 10.1146/annurev.neuro.051508.135409

Cui, G., Jun, S. B., Jin, X., Pham, M. D., Vogel, S. S., Lovinger, D. M., et al. (2013). Concurrent activation of striatal direct and indirect pathways during action initiation. Nature 494, 238-242. doi: 10.1038/nature11846

Dosenbach, N. U., Fair, D. A., Cohen, A. L., Schlaggar, B. L., and Petersen, S. E. (2008). A dual-networks architecture of top-down control. Trends Cogn. Sci. 12, 99-105. doi: 10.1016/j.tics.2008.01.001

Dosenbach, N. U., Fair, D. A., Miezin, F. M., Cohen, A. L., Wenger, K. K., Dosenbach, R. A., et al. (2007). Distinct brain networks for adaptive and stable task control in humans. Proc. Natl. Acad. Sci. U.S.A. 104, 11073-11078. doi: 10.1073/pnas.0704320104

Draganski, B., Kherif, F., Kloppel, S., Cook, P. A., Alexander, D. C., Parker, G. J., et al. (2008). Evidence for segregated and integrative connectivity patterns in the human Basal Ganglia. J. Neurosci. 28, 7143-7152. doi: 10.1523/JNEUROSCI.1486-08.2008

Ebisch, S. J., Mantini, D., Romanelli, R., Tommasi, M., Perrucci, M. G., Romani, G. L., et al. (2013). Long-range functional interactions of anterior insula and medial frontal cortex are differently modulated by visuospatial and inductive reasoning tasks. Neuroimage 78, 426-438. doi: 10.1016/j.neuroimage.2013.04.058

Fox, M. D., Snyder, A. Z., Vincent, J. L., Corbetta, M., Van Essen, D. C., and Raichle, M. E. (2005). The human brain is intrinsically organized into dynamic, anticorrelated functional networks. Proc. Natl. Acad. Sci. U.S.A. 102, 9673-9678. doi: 10.1073/pnas.0504136102

Francois-Brosseau, F. E., Martinu, K., Strafella, A. P., Petrides, M., Simard, F., and Monchi, O. (2009). Basal ganglia and frontal involvement in self-generated and externally-triggered finger movements in the dominant and non-dominant hand. Eur. J. Neurosci. 29, 1277-1286. doi: 10.1111/j.1460-9568.2009.06671.x

Georgiou, N., Iansek, R., Bradshaw, J. L., Phillips, J. G., Mattingley, J. B., and Bradshaw, J. A. (1993). An evaluation of the role of internal cues in the pathogenesis of parkinsonian hypokinesia. Brain 116(Pt 6),1575-1587. doi: 10.1093/brain/116.6.1575

Gottlich, M., Munte, T. F., Heldmann, M., Kasten, M., Hagenah, J., and Kramer, U. M. (2013). Altered resting state brain networks in Parkinson's disease. PLoS ONE 8:e77336. doi: 10.1371/journal.pone.0077336

Greicius, M. D., Krasnow, B., Reiss, A. L., and Menon, V. (2003). Functional connectivity in the resting brain: a network analysis of the default mode hypothesis. Proc. Natl. Acad. Sci. U.S.A. 100, 253-258. doi: 10.1073/pnas.0135058100

Haber, S. N. (2003). The primate basal ganglia: parallel and integrative networks. J. Chem. Neuroanat. 26, 317-330. doi: 10.1016/j.jchemneu.2003.10.003

Hagmann, P., Cammoun, L., Gigandet, X., Meuli, R., Honey, C. J., Wedeen, V. J., et al. (2008). Mapping the structural core of human cerebral cortex. PLoS Biol. 6:e159. doi: 10.1371/journal.pbio.0060159

He, Y., and Evans, A. (2010). Graph theoretical modeling of brain connectivity. Curr. Opin. Neurol. 23, 341-350. doi: 10.1097/WCO.0b013e32833aa567

Hoehn, M. M., and Yahr, M. D. (1967). Parkinsonism: onset, progression and mortality. Neurology 17, 427-442. doi: 10.1212/WNL.17.5.427

Howe, M. W., Tierney, P. L., Sandberg, S. G., Phillips, P. E., and Graybiel, A. M. (2013). Prolonged dopamine signalling in striatum signals proximity and value of distant rewards. Nature 500, 575-579. doi: 10.1038/nature12475

Huettel, S. A., Misiurek, J., Jurkowski, A. J., and McCarthy, G. (2004). Dynamic and strategic aspects of executive processing. Brain Res. 1000, 78-84. doi: 10.1016/j.brainres.2003.11.041

Hughes, A. J., Ben-Shlomo, Y., Daniel, S. E., and Lees, A. J. (1992). What features improve the accuracy of clinical diagnosis in Parkinson's disease: a clinicopathologic study. Neurology 42, 1142-1146. doi: 10.1212/WNL.42.6.1142

Isomura, Y., Takekawa, T., Harukuni, R., Handa, T., Aizawa, H., Takada, M., et al. (2013). Reward-modulated motor information in identified striatum neurons. J. Neurosci. 33, 10209-10220. doi: 10.1523/JNEUROSCI.0381-13.2013

Jacobson, S., and Marcus, E. M. (2007). Neuroanatomy for the Neuroscientist. New York, NY: Springer.

Joshua, M., Adler, A., and Bergman, H. (2009). The dynamics of dopamine in control of motor behavior. Curr. Opin. Neurobiol. 19, 615-620. doi: 10.1016/j.conb.2009.10.001

Kiesel, A., Steinhauser, M., Wendt, M., Falkenstein, M., Jost, K., Philipp, A. M., et al. (2010). Control and interference in task switching-a review. Psychol. Bull. 136, 849-874. doi: 10.1037/a0019842

Kitzbichler, M. G., Henson, R. N., Smith, M. L., Nathan, P. J., and Bullmore, E. T. (2011). Cognitive effort drives workspace configuration of human brain functional networks. J. Neurosci. 31, 8259-8270. doi: 10.1523/JNEUROSCI.044011.2011

Lago-Fernandez, L. F., Huerta, R., Corbacho, F., and Siguenza, J. A. (2000). Fast response and temporal coherent oscillations in small-world networks. Phys. Rev. Lett. 84, 2758-2761. doi: 10.1103/PhysRevLett.84.2758

Latora, V., and Marchiori, M. (2001). Efficient behavior of small-world networks. Phys. Rev. Lett. 87:198701. doi: 10.1103/PhysRevLett.87.198701

Leh, S. E., Chakravarty, M. M., and Ptito, A. (2008). The connectivity of the human pulvinar: a diffusion tensor imaging tractography study. Int. J. Biomed. Imaging 2008:789539. doi: 10.1155/2008/789539

Leh, S. E., Ptito, A., Chakravarty, M. M., and Strafella, A. P. (2007). Fronto-striatal connections in the human brain: a probabilistic diffusion tractography study. Neurosci. Lett. 419, 113-118. doi: 10.1016/j.neulet.2007.04.049

Lehericy, S., Ducros, M., Krainik, A., Francois, C., Van de Moortele, P. F., Ugurbil, K., et al. (2004). 3-D diffusion tensor axonal tracking shows distinct SMA and pre-SMA projections to the human striatum. Cereb. Cortex 14, 1302-1309. doi: 10.1093/cercor/bhh091

Lewis, S. J., Dove, A., Robbins, T. W., Barker, R. A., and Owen, A. M. (2003). Cognitive impairments in early Parkinson's disease are accompanied by reductions in activity in frontostriatal neural circuitry. J. Neurosci. 23, 6351-6356.

Litvan, I., Mohr, E., Williams, J., Gomez, C., and Chase, T. N. (1991). Differential memory and executive functions in demented patients with Parkinson's 
and Alzheimer's disease. J. Neurol. Neurosurg. Psychiatr. 54, 25-29. doi: 10.1136/jnnp.54.1.25

Mars, R. B., Klein, M. C., Neubert, F. X., Olivier, E., Buch, E. R., Boorman, E. D., et al. (2009). Short-latency influence of medial frontal cortex on primary motor cortex during action selection under conflict. J. Neurosci. 29, 6926-6931. doi: 10.1523/JNEUROSCI.1396-09.2009

Martinu, K., Degroot, C., Madjar, C., Strafella, A. P., and Monchi, O. (2012). Levodopa influences striatal activity but does not affect cortical hyper-activity in Parkinson's disease. Eur. J. Neurosci. 35, 572-583. doi: 10.1111/j.14609568.2011.07979.x

Masuda, N., and Aihara, K. (2004). Global and local synchrony of coupled neurons in small-world networks. Biol. Cybern. 90, 302-309. doi: 10.1007/s00422-0040471-9

McFarland, N. R., and Haber, S. N. (2002). Thalamic relay nuclei of the basal ganglia form both reciprocal and nonreciprocal cortical connections, linking multiple frontal cortical areas. J. Neurosci. 22, 8117-8132.

McHaffie, J. G., Stanford, T. R., Stein, B. E., Coizet, V., and Redgrave, P. (2005). Subcortical loops through the basal ganglia. Trends Neurosci. 28, 401-407. doi: 10.1016/j.tins.2005.06.006

Monchi, O., Petrides, M., Doyon, J., Postuma, R. B., Worsley, K., and Dagher, A. (2004). Neural bases of set-shifting deficits in Parkinson's disease. J. Neurosci. 24, 702-710. doi: 10.1523/JNEUROSCI.4860-03.2004

Monchi, O., Petrides, M., Mejia-Constain, B., and Strafella, A. P. (2007). Cortical activity in Parkinson's disease during executive processing depends on striatal involvement. Brain 130, 233-244. doi: 10.1093/brain/awl326

Monchi, O., Petrides, M., Petre, V., Worsley, K., and Dagher, A. (2001). Wisconsin Card Sorting revisited: distinct neural circuits participating in different stages of the task identified by event-related functional magnetic resonance imaging. J. Neurosci. 21, 7733-7741.

Nagano-Saito, A., Habak, C., Mejia-Constain, B., Degroot, C., Monetta, L., Jubault, T., et al. (2013). Effect of mild cognitive impairment on the patterns of neural activity in early Parkinson's disease. Neurobiol. Aging 35, 223-231. doi: 10.1016/ j.neurobiolaging.2013.06.025

Nagano-Saito, A., Leyton, M., Monchi, O., Goldberg, Y. K., He, Y., and Dagher, A. (2008). Dopamine depletion impairs frontostriatal functional connectivity during a set-shifting task. J. Neurosci. 28, 3697-3706. doi: 10.1523/JNEUROSCI.3921-07.2008

Nathaniel-James, D. A., and Frith, C. D. (2002). The role of the dorsolateral prefrontal cortex: evidence from the effects of contextual constraint in a sentence completion task. Neuroimage 16, 1094-1102. doi: 10.1006/nimg.2002.1167

Parent, A., and Hazrati, L. N. (1995). Functional anatomy of the basal ganglia. I. The cortico-basal ganglia-thalamo-cortical loop. Brain Res. Brain Res. Rev. 20, 91-127. doi: 10.1016/0165-0173(94)00007-C

Picard, N., and Strick, P. L. (2001). Imaging the premotor areas. Curr. Opin. Neurobiol. 11, 663-672. doi: 10.1016/S0959-4388(01)00266-5

Power, J. D., Cohen, A. L., Nelson, S. M., Wig, G. S., Barnes, K. A., Church, J. A., et al. (2011). Functional network organization of the human brain. Neuron 72 , 665-678. doi: 10.1016/j.neuron.2011.09.006

Sakagami, M., and Pan, X. (2007). Functional role of the ventrolateral prefrontal cortex in decision making. Curr. Opin. Neurobiol. 17, 228-233. doi: 10.1016/j.conb.2007.02.008

Sakagami, M., Tsutsui, K., Lauwereyns, J., Koizumi, M., Kobayashi, S., and Hikosaka, O. (2001). A code for behavioral inhibition on the basis of color, but not motion, in ventrolateral prefrontal cortex of macaque monkey. J. Neurosci. 21, 4801-4808.

Samii, A., Nutt, J. G., and Ransom, B. R. (2004). Parkinson's disease. Lancet 363, 1783-1793. doi: 10.1016/S0140-6736(04)16305-8

Schultz, W. (1998). Predictive reward signal of dopamine neurons. J. Neurophysiol. $80,1-27$.
Seamans, J. K., and Yang, C. R. (2004). The principal features and mechanisms of dopamine modulation in the prefrontal cortex. Prog. Neurobiol. 74, 1-58. doi: 10.1016/j.pneurobio.2004.05.006

Siegel, M., Engel, A. K., and Donner, T. H. (2011). Cortical network dynamics of perceptual decision-making in the human brain. Front. Hum. Neurosci. 5:21. doi: 10.3389/fnhum.2011.00021

Skidmore, F., Korenkevych, D., Liu, Y., He, G., Bullmore, E., and Pardalos, P. M. (2011). Connectivity brain networks based on wavelet correlation analysis in Parkinson fMRI data. Neurosci. Lett. 499, 47-51. doi: 10.1016/j.neulet.2011.05.030

Sporns, O., Tononi, G., and Edelman, G. M. (2000). Theoretical neuroanatomy: relating anatomical and functional connectivity in graphs and cortical connection matrices. Cereb. Cortex 10, 127-141. doi: 10.1093/cercor/ 10.2.127

Surmeier, D. J., Plotkin, J., and Shen, W. (2009). Dopamine and synaptic plasticity in dorsal striatal circuits controlling action selection. Curr. Opin. Neurobiol. 19, 621-628. doi: 10.1016/j.conb.2009.10.003

Takada, M., Tokuno, H., Nambu, A., and Inase, M. (1998). Corticostriatal input zones from the supplementary motor area overlap those from the contrarather than ipsilateral primary motor cortex. Brain Res. 791, 335-340. doi: 10.1016/S0006-8993(98)00198-X

Toro, R., Fox, P. T., and Paus, T. (2008). Functional coactivation map of the human brain. Cereb. Cortex 18, 2553-2559. doi: 10.1093/cercor/bhn014

van Schouwenburg, M. R., den Ouden, H. E., and Cools, R. (2010). The human basal ganglia modulate frontal-posterior connectivity during attention shifting. J. Neurosci. 30, 9910-9918. doi: 10.1523/JNEUROSCI.1111-10.2010

Watts, D. J., and Strogatz, S. H. (1998). Collective dynamics of "small-world" networks. Nature 393, 440-442. doi: 10.1038/30918

Werheid, K., Koch, I., Reichert, K., and Brass, M. (2007). Impaired self-initiated task preparation during task switching in Parkinson's disease. Neuropsychologia 45, 273-281. doi: 10.1016/j.neuropsychologia.2006.07.007

Wu, T., Wang, L., Chen, Y., Zhao, C., Li, K., and Chan, P. (2009). Changes of functional connectivity of the motor network in the resting state in Parkinson's disease. Neurosci. Lett. 460, 6-10. doi: 10.1016/j.neulet.2009. 05.046

Wu, T., Wang, L., Hallett, M., Chen, Y., Li, K., and Chan, P. (2011). Effective connectivity of brain networks during self-initiated movement in Parkinson's disease. Neuroimage 55, 204-215. doi: 10.1016/j.neuroimage.2010. 11.074

Yeterian, E. H., and Pandya, D. N. (1995). Corticostriatal connections of extrastriate visual areas in rhesus monkeys. J. Comp. Neurol. 352, 436-457. doi: $10.1002 /$ cne. 903520309

Conflict of Interest Statement: The authors declare that the research was conducted in the absence of any commercial or financial relationships that could be construed as a potential conflict of interest.

Received: 01 April 2014; accepted: 12 June 2014; published online: 08 July 2014. Citation: Nagano-Saito A, Martinu Kand Monchi O (2014) Function of basal ganglia in bridging cognitive and motor modules to perform an action. Front. Neurosci. 8:187. doi: $10.3389 /$ fnins.2014.00187

This article was submitted to Decision Neuroscience, a section of the journal Frontiers in Neuroscience.

Copyright $\odot 2014$ Nagano-Saito, Martinu and Monchi. This is an open-access article distributed under the terms of the Creative Commons Attribution License (CC BY). The use, distribution or reproduction in other forums is permitted, provided the original author(s) or licensor are credited and that the original publication in this journal is cited, in accordance with accepted academic practice. No use, distribution or reproduction is permitted which does not comply with these terms. 